\title{
Quasi-isometric and Quasi-conformal Development of Triangulated Surfaces for Computerized Tomography
}

\author{
Eli Appleboim ${ }^{1}$, Emil Saucan ${ }^{1}$, Yehoshua Y. Zeevi ${ }^{1}$, and Ofir Zeitoun ${ }^{2}$ \\ ${ }^{1}$ Electrical Engineering Department, Technion, Haifa, Israel \\ \{eliap, semil, zeevi\}@tx.technion.ac.il \\ ${ }^{2}$ FRS Ltd., Rosh HaAyin, Israel \\ ofir.zeitoun@gmail.com
}

\begin{abstract}
In this paper we present a simple method for minimal distortion development of triangulated surfaces for mapping and imaging. The method is based on classical results of F. Gehring and Y. Väisälä regarding the existence of quasi-conformal and quasi-isometric mappings between Riemannian manifolds. A random starting triangle version of the algorithm is presented. A curvature based version is also applicable. In addition the algorithm enables the user to compute the maximal distortion errors. Moreover, the algorithm makes no use to derivatives, hence it is suitable for analysis of noisy data. The algorithm is tested on data obtained from real CT images of the human brain cortex.
\end{abstract}

\section{Introduction}

Two-dimensional representation of three-dimensional object scan, are encountered in image processing. Medical imaging, computer aided design and reverse engineering are three of the important examples. For example, one should be able to present the three-dimensional MRI/CT scans of the brain cortex, as a (set of) two-dimensional images. Yet, in order to do so in a meaningful manner, so that diagnosis will be accurate, it is essential that the geometric distortion, in terms of change of angles and lengths, caused by this representation will be minimal. In computer graphic, this problem is sometimes refereed to as, surface/freeform parameterization. Applications are also found in texture mapping, surface re-meshing, surface compression, and others.

In most cases, since the surfaces considered are not isometric to the plane, one cannot expect a zero distortion solution. Yet, a reasonable solution to this problem is given by conformal maps (i.e. maps that preserve angles). This is done by mapping the surface conformally to the (complex) plane. Since this cannot be achieved in a global way, all solutions are local.

If one is willing to absorb some bounded amount of distortion then quasiisometric/quasi-conformal maps (i.e. maps that are almost isometries/conformal - the precise definition will follow in Section 2) will also suffice.

As in many other cases, the tradeoff is between simplicity/cost of implementation on one hand and accuracy on the other. Common to all proposed solutions is 
the fact, which really cannot be avoided because of the inevitable distortion, that the more locally one is willing to focus, the more accurate the results become.

\subsection{Related Works}

As stated above, the problem of minimal distortion flattening of surfaces attracted, in recent years, a great attention and interest, due to its wide range of applications.

In this section we briefly review some of the methods that were proposed for dealing with this problem.

Variational Methods. Haker et al. ([8], [9]) introduced the use of a variational method for conformal flattening of CT/MRI 3-D scans of the brain/colon for the purpose of medical imaging. The method is essentially based on solving Dirichlet problem for the Laplace-Beltrami operator $\triangle u=0$ on a given surface $\Sigma$, with boundary conditions on $\partial \Sigma$. A solution to this problem is a harmonic (thus conformal) map from the surface to the (complex) plane. The solution suggested in [8] and [9] is a PL (piecewise linear) approximation of the smooth solution, achieved by solving a proper system of linear equations.

Circle Packing. Hurdal et al. ([10]) attempt to obtain such a conformal map by using circle packing. This relies on the ability to approximate conformal structure on surfaces by circle packings. The authors use this method for MRI brain images and conformally map them to the three possible models of geometry in dimension 2 (i.e. the 2-sphere, the Euclidian plane and the Hyperbolic plane). Yet, the method is applicable for surfaces which are topologically equivalent to a disk whereas the brain cortex surface is not. This means that there is a point of the brain (actually a neighborhood of a point), which will not map conformally to the plane, and in this neighborhood the dilatation will be infinitely large. An additional problem arises due to the necessary assumption that the surface triangulation is homogeneous in the sense that all triangles are equilateral. Such triangulations are seldom attainable.

Holomorphic 1-Forms. Gu et al. ([6], [7], [5]) are using holomorphic 1-forms in order to compute global conformal structure of a smooth surface of arbitrary genus given as a triangulated mesh. holomorphic 1-forms are differential forms (differential operators) on smooth manifolds, which among other things can depict conformal structures. The actual computation is done via computing homology/co-homology bases for the first homology/co-homology groups of the surface, $H_{1}, H^{1}$ respectively. This method indeed yields a global conformal structure hence, a conformal parameterization for the surface however, computing homology basis is extremely time consuming.

Angle Methods. In [12] Sheffer et al. parameterize surfaces via an angle based method in a way that minimizes angle distortion while flattening. However, the surfaces are assumed to be approximated by cone surfaces, i.e. surfaces that are composed from cone-like neighborhoods. 
To summarize, all the methods described above compute only approximation to conformal mappings, therefore producing only quasi-conformal mappings, with no precise estimates on the dilatation.

In this paper we propose yet another solution to this problem. The proposed method relies on theoretical results obtained by Gehring and Väisalä in the 1960 s ([4]). They were studying the existence of quasi-conformal maps between Riemannian manifolds. The basic advantages of this method resides in its simplicity, in setting, implementation and its speed. Additional advantage is that it is possible guarantee not to have distortion above a predetermined bound, which can be as small as desired, with respect to the amount of localization one is willing to pay (and, in the case of triangulated surfaces, to the quality of the given mesh). The suggested algorithm is best suited to cases where the surface is complex (high and non-constant curvature) such as brain cortex/colon wrapping, or of large genus such as skeleta, proteins, etc. Moreover, since toghether with the angular dilatation, both length and area distortions are readily computable, the algorithm is ideally suited for applications in Oncology, where such measurements are highly relevant.

The paper is organized as follows, in the next section we introduce the theoretical background, regarding the fundamental work of Gehring and Väisalä. Afterwards we describe our algorithm for surface flattening, based on their ideas. In Section 4 we present some experimental results of this scheme and in Section 5 we discuss possible extensions of this study. We include two appendices regarding some classical notions in quasi-conformal mapping theory and the definition of the essential supremum, respectively.

\section{Theoretical Background}

\subsection{Basic Definitions}

Definition 1. Let $D \subset \mathbb{R}^{3}$ be a domain. A homeomorphism $f: D \rightarrow \mathbb{R}^{3}$ is called a quasi-isometry (or a bi-lipschitz mapping), if there exists $1 \leq C<\infty$, such that

$$
\frac{1}{C}\left|p_{1}-p_{2}\right| \leq\left|f\left(p_{1}\right)-f\left(p_{2}\right)\right|<C\left|p_{1}-p_{2}\right|, \text { for all } p_{1}, p_{2} \in D .
$$

$C(f)=\min \{C \mid f$ is a quasi - isometry $\}$ is called the minimal distortion of $f($ in $D)$.

Note. For the case of surface embedded in $\mathbb{R}^{3}$ distances are the induced intrinsic distances on the surfaces.

Remark 1. If $f$ is a quasi-isometry then $K_{I}(f) \leq C(f)^{2}$ and $K_{O}(f) \leq C(f)^{2}$ where $K_{I}(f), K_{O}(f)$ represent the inner, respective outer dilatation of $f$, (see Appendix 1). It follows that any quasi-isometry is a quasi-conformal mapping (while - evidently - not every quasi-conformal mapping is a quasi-isometry). Quasi-conformal is the same as quasi-isometry where distances are replaced by angles between tangent vectors. 
Definition 2. Let $S \subset \mathbb{R}^{3}$ be a connected set. $S$ is called admissible (see Fig. 1) iff for any $p \in S$, there exists a quasi-isometry $i_{p}$ such that for any $\varepsilon>0$ there exists a neighbourhood $U_{p} \subset \mathbb{R}^{3}$ of $p$, such that $i_{p}: U_{p} \rightarrow \mathbb{R}^{3}$ and $i_{p}\left(S \cap U_{p}\right)=D_{p} \subset \mathbb{R}^{2}$, where $D_{p}$ is a domain and such that $C\left(i_{p}\right)$ satisfies:

(i) $\sup _{p \in S} C\left(i_{p}\right)<\infty$

and

(ii) ess $\sup _{p \in S} C\left(i_{p}\right)<1+\varepsilon$

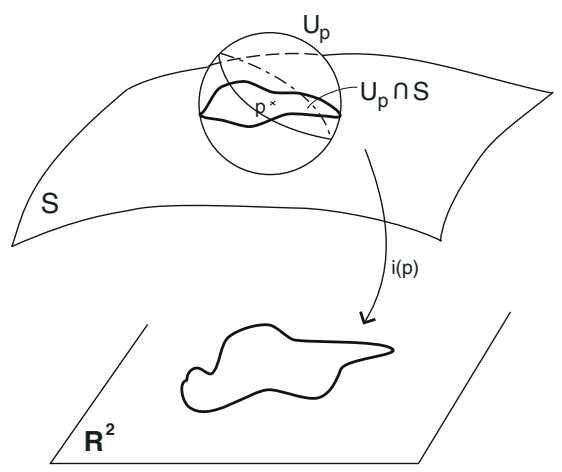

Fig. 1. An Admissible Surface

\subsection{The Projection Map}

Let $S$ be a surface, $\overline{\boldsymbol{n}}$ be a fixed unitary vector, and $p \in S$. Let $V \simeq D^{2}$, $D^{2}=\left\{x \in \mathbb{R}^{2} \mid\|x\| \leq 1\right\}$ be a disk neighbourhood of $p$. Moreover, suppose that for any $q_{1}, q_{2} \in S$, the acute angle $\measuredangle\left(q_{1} q_{2}, \overline{\boldsymbol{n}}\right) \geq \alpha$ (see Figure 2$)$. We refer to the last condition as the Geometric Condition or Gehring Condition.

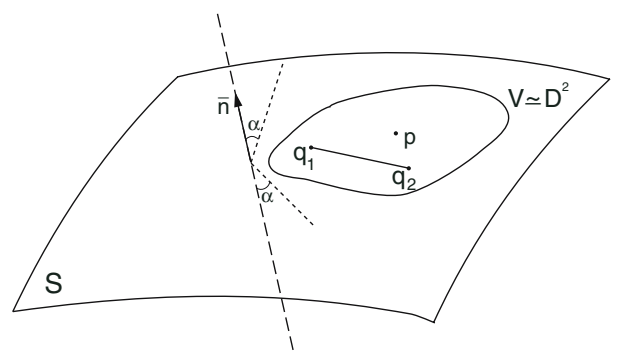

Fig. 2. The Geometric Condition 
Then for any $x \in V$ there is a unique representation of the following form:

$$
x=q_{x}+u \boldsymbol{n}
$$

where $q_{x}$ lies on the plane through $p$ which is orthogonal to $\boldsymbol{n}$ and $u \in \mathbb{R}$.

Define:

$$
\operatorname{Pr}(x)=q_{x} .
$$

Note: $\boldsymbol{n}$ need not be the normal vector to $S$ at $p$.

By [4], Section 4.3 and Lemma 5.1, we have that for any $p_{1}, p_{2} \in S$ and any $a \in \mathbb{R}_{+}$the following inequalities hold:

$$
\frac{a}{A}\left|p_{1}-p_{2}\right| \leq\left|\operatorname{Pr}\left(p_{1}\right)-\operatorname{Pr}\left(p_{2}\right)\right| \leq A\left|p_{1}-p_{2}\right|
$$

where

$$
A=\frac{1}{2}\left[(a \csc \alpha)^{2}+2 a+1\right]^{2}+\frac{1}{2}\left[(a \csc \alpha)^{2}-2 a+1\right]^{2} .
$$

In particular for $a=1$ we get that

$$
C(f) \leq \cot \alpha+1
$$

and

$$
K(f) \leq\left(\left(\frac{1}{2}(\cot \alpha)^{2}+4\right)^{\frac{1}{2}}+\frac{1}{2} \cot \alpha\right)^{\frac{3}{2}} \leq(\cot \alpha+1)^{\frac{3}{2}}
$$

where

$$
K(f)=\max \left(K_{O}(f), K_{I}(f)\right)
$$

is the maximal dilatation of $f$.

Hence we have thus obtained the desired quasi-isometry, $\operatorname{Pr}$, having maximal dilatation,

$$
C(f) \leq \cot \alpha+1
$$

The Geometric Condition. From the discussion above we conclude that $S \subset$ $\mathbb{R}^{3}$ is an admissible surface if for any $p \in S$ there exists $\boldsymbol{n}_{p}$ such that for any $\varepsilon>0$, there exists $U_{p} \simeq D^{2}$, such that for any $q_{1}, q_{2} \in U_{p}$ the acute angle $\measuredangle\left(q_{1} q_{2}, \boldsymbol{n}_{p}\right) \geq \alpha$, where

$$
\text { (i) } \inf _{p \in S} \alpha_{p}>0
$$

and

$$
\text { (ii) ess } \inf _{p \in S} \alpha_{p}<\frac{\pi}{2}-\varepsilon
$$

Example 1. Any surface in $S \in \mathbb{R}^{3}$ that admits a well-defined continuous turning tangent plane at any point $p \in S$ is admissible. 


\section{The Algorithm}

We will present in this section the algorithm that is used for obtaining a quasiisometric (flat) representation of a given surface.

First assume the surface is equipped with some triangulation $T$. Let $N_{p}$ stand for the normal vector to the surface at a point $p$ on the surface.

Second, a triangle $\Delta$, of the triangulation must be chosen. We will project a patch of the surface quasi-isometrically onto the plane included in $\Delta$. This patch will be called the patch of $\Delta$, and it will consists of at least one triangle, $\Delta$ itself. There are two possibilities to chose $\Delta$, one is in a random manner and the other is based on curvature considerations. We will refer to both ways later. For the moment assume $\Delta$ was somehow chosen. After $\Delta$ is (trivially) projected onto itself we move to its neighbors. Suppose $\Delta^{\prime}$ is a neighbor of $\Delta$ having edges $e_{1}$, $e_{2}, e_{3}$, where $e_{1}$ is the edge common to both $\Delta$ and $\Delta^{\prime}$.

We will call $\Delta^{\prime}$ Gehring compatible w.r.t $\Delta$, if the maximal angle between $e_{2}$ or $e_{3}$ and $N_{\Delta}$ (the normal vector to $\Delta$ ), is greater then a predefined measure suited to the desired predefined maximal allowed distortion, i.e. $\max \left\{\varphi_{1}, \varphi_{2}\right\} \geq \alpha$, where $\varphi_{1}=\measuredangle\left(e_{2}, N_{\Delta}\right), \varphi_{2}=\measuredangle\left(e_{3}, N_{\Delta}\right)$.

We will project $\Delta^{\prime}$ orthogonally onto the plane included in $\Delta$ and insert it to the patch of $\Delta$, iff it is Gehring compatible w.r.t $\Delta$.

We keep adding triangles to the patch of $\Delta$ moving from an added triangle to its neighbors (of course) while avoiding repetitions, till no triangles can be added.

If by this time all triangles where added to the patch we have completed constructing the mapping. Otherwise, chose a new triangle that has not been projected yet, to be the starting triangle of a new patch. A pseudocode for this procedure can be easily written.

Remark 2. There are two ways for choosing a base triangle for each patch. One is by taking a triangle which the sum of the (magnitude of) curvatures of its vertices is minimal, and the other one is by letting the user choose a triangle for each new patch.

Remark 3. One should keep in mind that the above given algorithm, as for any other flattening method, is local. Indeed, in a sense the (proposed) algorithm gives a measure of "globality" of this intrinsically local process.

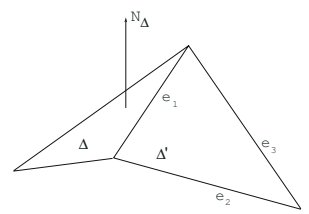

Fig. 3. Gehring Compatible Triangles 
Remark 4. Our algorithm is best suited for highly folded surfaces, because of its intrinsic locality, on the one hand, and computational simplicity, on the other. However, on "quasi-developable" surfaces (i.e. surfaces that are almost cylindrical or conical) the algorithm behaves similar to other algorithms, with practically identical results).

\section{Experimental Results}

We now proceed to present some experimental results obtained by applying the proposed algorithm, both on synthetic surfaces and on data obtained from actual CT scans.

In each of the examples both the input surface and a flattened representation of some patch are shown. Details about mesh resolution as well as flattening distortion are also provided. The number of patches needed in order to flatten the surface is also given. In all images, the small rectangle shown on the surface represents a base triangle for the flattened patch.

The algorithm was implemented in two versions, or more precisely two possible ways of processing, automatic versus user defined.

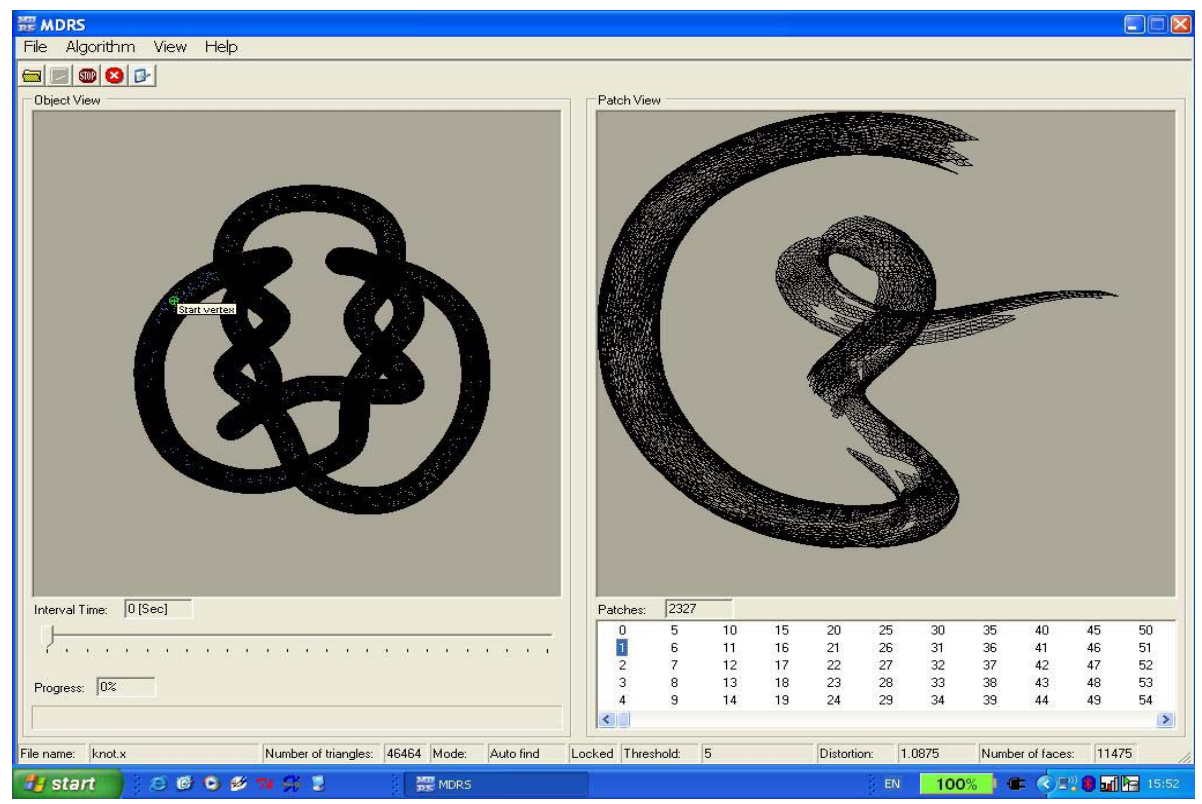

Fig. 4. Knotted Torus: The resolution of the model is of 46,464 triangles. The patch contains 11, 475 triangles. The low selected value of $\alpha$ is $5^{\circ}$, giving a dilatation equal to 1.0875 . Due to the low Gauss curvature of the embedding of the knot, two such patches cover most of the flattened surface. 
1. Automatic means that the triangles serving as base points for the patches to be flattened are chosen automatically according to curvature, as stated in Remark 3.1. The discrete curvature measure employed is that of angular defect, due to its simplicity and high reliability (see [13]).

2. User defined means that at each stage the user chooses a base triangle for some new patch.

The colored area in each of the images represents the patch being flattened.

Experiments have shown that results of the automatic process are similar, in terms of the dilatation, to those obtained from the user defined process yet, in order to flatten entire surface in the user defined method one needs in average 25 percent more patches.

\section{Concluding Remarks and Future Study}

In this paper we presented a new algorithm for flattened presentation of polyhedral meshes, with minimal dilatation while flattening is done. The algorithm is based upon the works of Gehring-Väisala and others concerning the existence of quasi-isometric/conformal/meromorphic mappings between Riemannian manifolds.

From the implementation results it is evident that this algorithm while being simple to program as well as efficient, also gives good flattening results and maintains small dilatations even in areas where curvature is large and good flattening is a challenging task. Moreover, since there is a simple way to assess the resulting dilatation, the algorithm was implemented in such a way that the user can set in advance an upper bound on the resulting dilatation.

An additional advantage of the presented algorithm is related to the fact that, contrary to some of the related studies, no use of derivatives is made. Consequently, the algorithm does not suffer from typical drawbacks of derivative computations like robustness, etc.

Moreover, since no derivatives are employed, no smoothness assumption about the surface to be flattened are made, which makes the algorithm presented herein ideal for use in cases where smoothness is questionable (to say the least).

The algorithm may be practical for applications where local yet, good analysis is required such as medical imaging with the emphasis on flattened representation of the brain and the colon (virtual colonoscopy) - see [1], [2]. Further study is currently undertaken.

The main issue for further investigation, remains the transition from local to global in a more precise fashion, i.e. how can one glue two neighbouring patches while keeping fixed bounded dilatation. (In more technical terms, this amounts to actually computing the holonomy map of the surface - see [14].)

Indeed, we may flatten the neighborhood of some vertex $u$ obtaining the flat image $I_{u}$ and the neighborhood of another vertex $v$ obtaining the image $I_{v}$ so that these two neighborhood have some intersection along the boundary yet, it will not be possible to adjust the resulting images to give one flat image $I_{u \cup v}$ of 
the union of these neighborhood, yet satisfy the quasi-isometric property. This too is also under current investigation.

\section{Acknowledgment}

Emil Saucan is supported by the Viterbi Postdoctoral Fellowship. Research is partly supported by the Ollendorf Minerva Center.

\section{References}

[1] Appleboim, E., Saucan E., and Zeevi, Y. Minimal-Distortion Mappings of Surfaces for Medical Imaging, Proceedings of VISAPP 2006, to appear.

[2] Appleboim, E., Saucan, E., and Zeevi, Y.Y. On Sampling and Reconstruction of Surfaces, Technion CCIT Report, 2006.

[3] Caraman, P. n-Dimensional Quasiconformal (QCf) Mappings, Editura Academiei Române, Bucharest, Abacus Press, Tunbridge Wells Haessner Publishing, Inc., Newfoundland, New Jersey, 1974.

[4] Gehring, W. F. and Väisälä, J. The coefficients of quasiconformality, Acta Math. 114, pp. 1-70, 1965.

[5] Gu, X. Wang, Y. and Yau, S. T. Computing Conformal Invariants: Period Matrices, Communications In Information and Systems, Vol. 2, No. 2, pp. 121-146, December 2003.

[6] Gu, X. and Yau, S. T. Computing Conformal Structure of Surfaces, Communications In Information and Systems, Vol. 2, No. 2, pp. 121-146, December 2002.

[7] Gu, X. and Yau, S. T. Global Conformal Surface Parameterization, Eurographics Symposium on Geometry Processing, 2003.

[8] Haker, S. Angenet, S. Tannenbaum, A. Kikinis, R. Non Distorting Flattening Maps and the 3-D visualization of Colon CT Images, IEEE Transauctions on Medical Imaging, Vol. 19, NO. 7, July 2000.

[9] Haker, S. Angenet, S. Tannenbaum, A. Kikinis, R. Sapiro, G. Halle, M. Conformal Surface Parametrization for Texture Mapping, IEEE Transauctions on Visualization and Computer Graphics, Vol. 6, NO. 2, June 2000.

[10] Hurdal, M. K., Bowers, P. L., Stephenson, K., Sumners, D. W. L., Rehm, K., Schaper. K., Rottenberg, D. A. Quasi Conformally Flat Mapping the Human Crebellum, Medical Image Computing and Computer-Assisted Intervention MICCAI'99, (C. Taylor and A. Colchester. eds), vol. 1679, Springer-Verlag, Berlin, 279-286, 1999.

[11] Stephenson, K. personal communication.

[12] Sheffer, A. de Stuler, E. Parametrization of Faceted Surfaces for Meshing Using Angle Based Flattening, Enginneering with Computers, vol. 17, pp. 326-337, 2001.

[13] Surazhsky, T., Magid, E., Soldea, O., Elber, G. and Rivlin E. A Comparison of Gaussian and Mean Curvatures Estimation Methods on Triangular Meshes, Proceedings of the IEEE International Conference on Robotics and Automation. Taipei, Taiwan, pp 1021-1026, September 2003.

[14] Thurston, W. Three-Dimensional Geometry and Topology, vol.1, (Edited by S. Levy), Princeton University Press, Princeton, N.J. 1997.

[15] Väisalä, J. Lectures on n-dimensional quasiconformal mappings, Lecture Notes in Mathematics 229, Springer-Verlag, Berlin - Heidelberg - New-York, 1971. 


\section{Appendix 1}

Let $D \subset \mathbb{R}^{3}$ be a domain, let $f: D \stackrel{\sim}{\rightarrow} f(D)$ and let $p \in D$.

We make the following notations:

$$
\begin{gathered}
L(p)=\limsup _{x \rightarrow p} \frac{|f(x)-f(p)|}{|x-p|} ; \\
l(p)=\liminf _{x \rightarrow p} \frac{|f(x)-f(p)|}{|x-p|} ; \\
J(p)=\limsup _{r \rightarrow 0} \frac{\operatorname{Vol}\left(f\left(\mathbb{B}^{3}(p, r)\right)\right)}{\operatorname{Vol}\left(\mathbb{B}^{3}(p, r)\right)} .
\end{gathered}
$$

$L(p), l(p)$ are called the maximal, respective minimal stretching, of $f$. If $f$ is differentiable then $J(p)=|\operatorname{Jacobian}(f)(p)|$.

Then, if $J>0$ and if $f$ is $A C L$ (see, e.g. [15]), then the maximal and minimal stretching can be defined as follows:

$$
\begin{gathered}
K_{I}(f)=\sqrt{\underset{p \in D}{\operatorname{ess} \sup \frac{J(p)}{l^{3}(p)}}} ; \\
K_{O}(f)=\sqrt{\underset{p \in D}{\operatorname{ess} \sup _{p} \frac{L^{3}(p)}{J(p)}} .}
\end{gathered}
$$

\section{Appendix 2}

The essential supremum of $f$ is the smallest number $a$ for which $f$ only exceeds $a$ on a set of measure zero. More formally, we have the following definition:

Definition 3. Let $(X, \mathcal{B}, \mu)$ be a measure space, let $f: X \rightarrow \mathbb{R}$, and let $a \in \mathbb{R}$. Define $\left.\left.M_{a}=\{x \mid f) x\right)>0\right\}$ and $A_{0}=\{a \in \mathbb{R} \mid \mu(x)=0\}$. Then:

$$
\operatorname{ess} \sup f=\inf A_{0} \text {. }
$$

(If $A_{0}=\emptyset$, then we define ess $\sup f=\infty$.)

Remark 5. In our case $\mu$ is the 2-dimensional Hausddorff measure (see, e.g. [3]). 


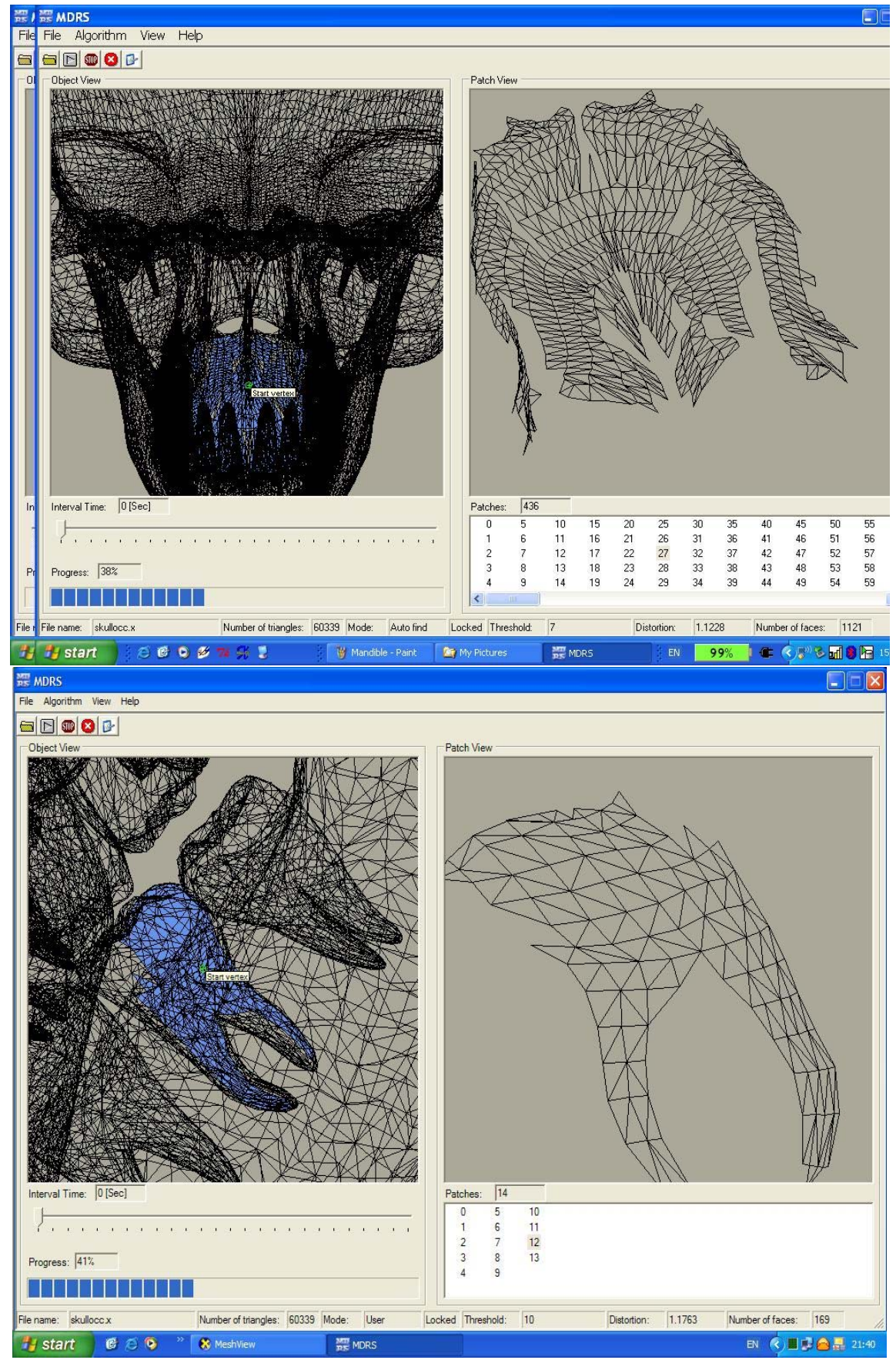

Fig. 5. Skull model: The resolution is of 60,339 triangles. Top: $\alpha=7^{\circ}$ and the dilatation is 1.1228. Bottom: $\alpha=10^{\circ}$ and the dilatation is 1.1763 . 


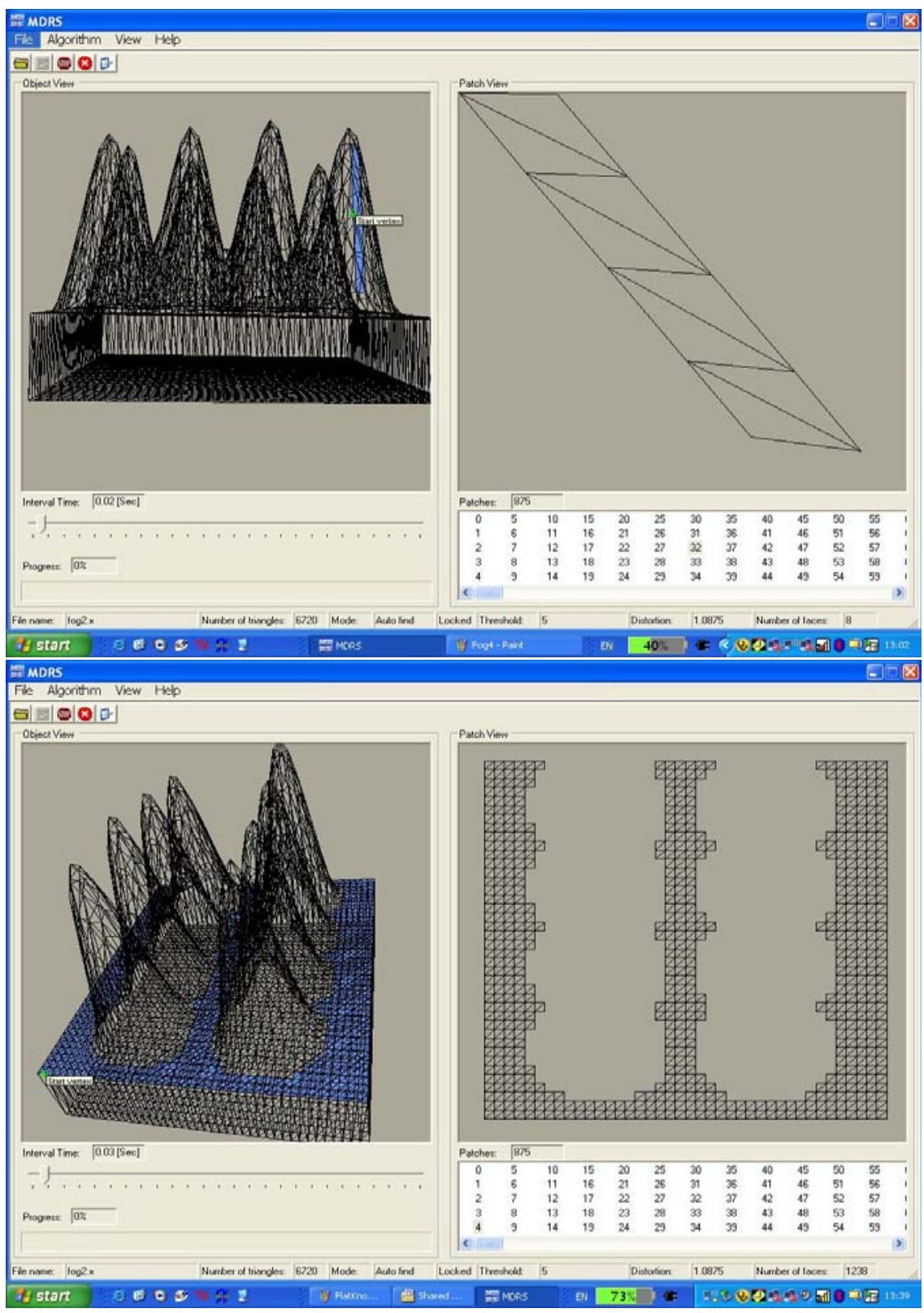

Fig. 6. A Analytic Surface: Observe the role of low Gauss curvature in producing large patches, even of genus higher then 0, (b). Here the resolution is 6720 triangles and $\alpha=5^{\circ}$. 


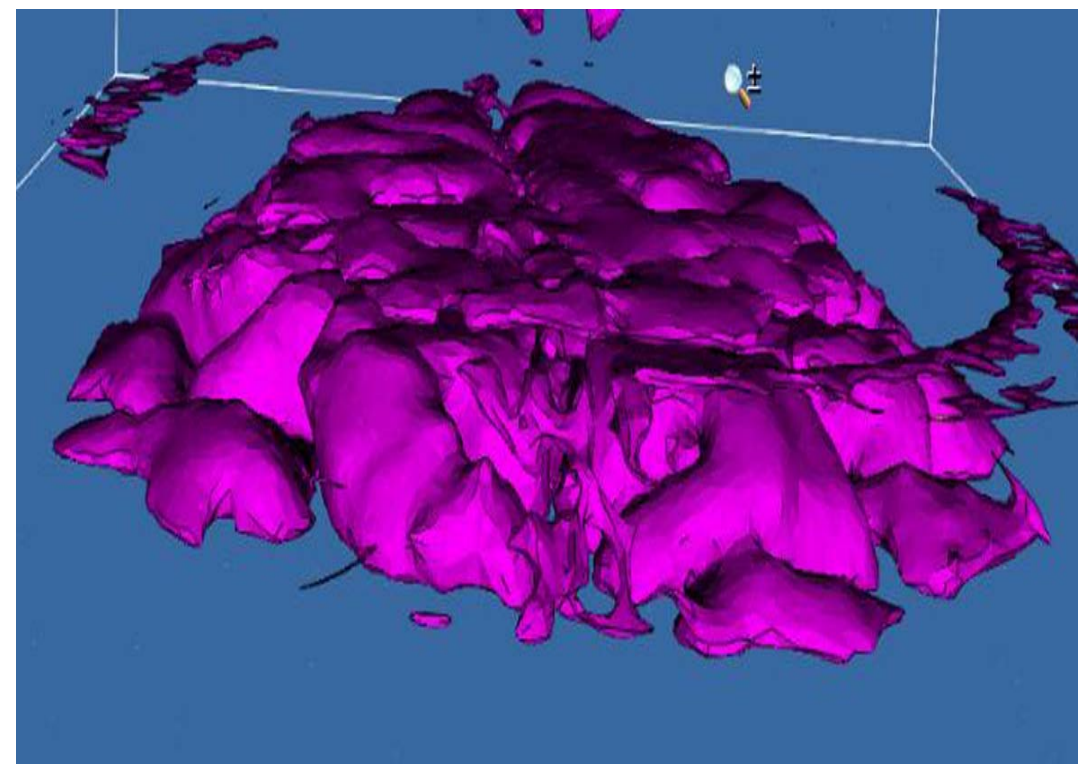

Fig. 7. Cerebral Cortex Flattening: The location of the cortical region selected for flattening in the previous figure

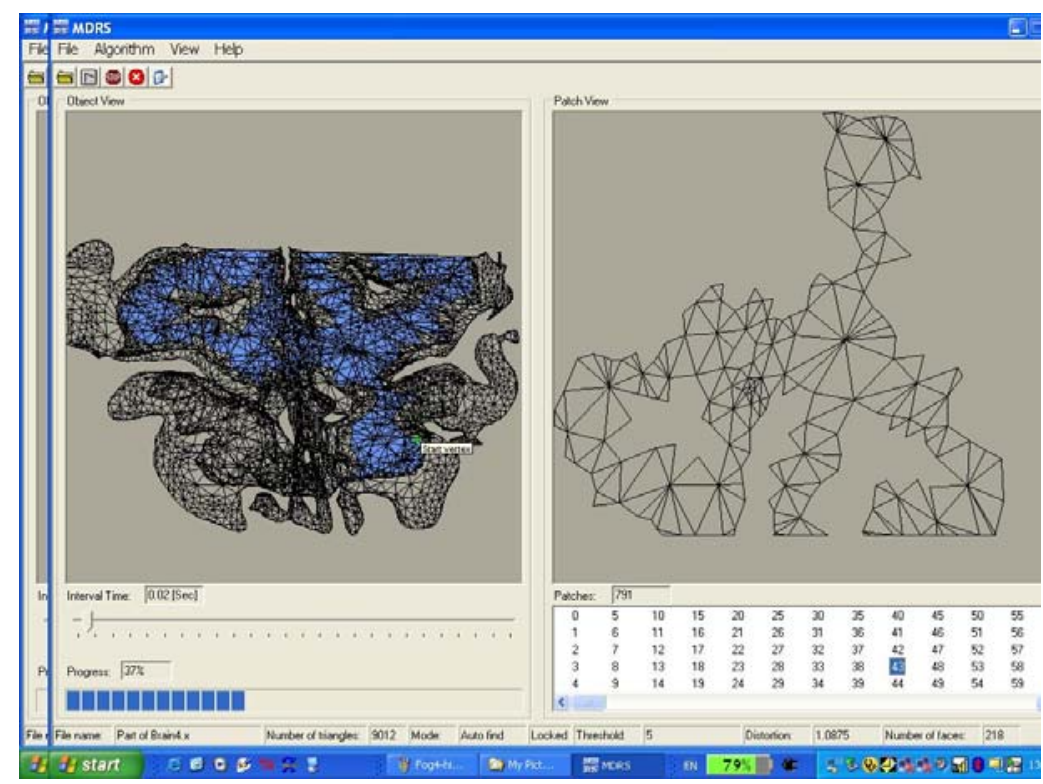

Fig. 8. Cerebral Cortex Flattening: Partial view of the parietal region. Observe that a non-simply connected patch is obtained. The resolution is 15.110 triangles, the angles chosen are $5^{\circ}$, producing dilatations of 1.0875 . 


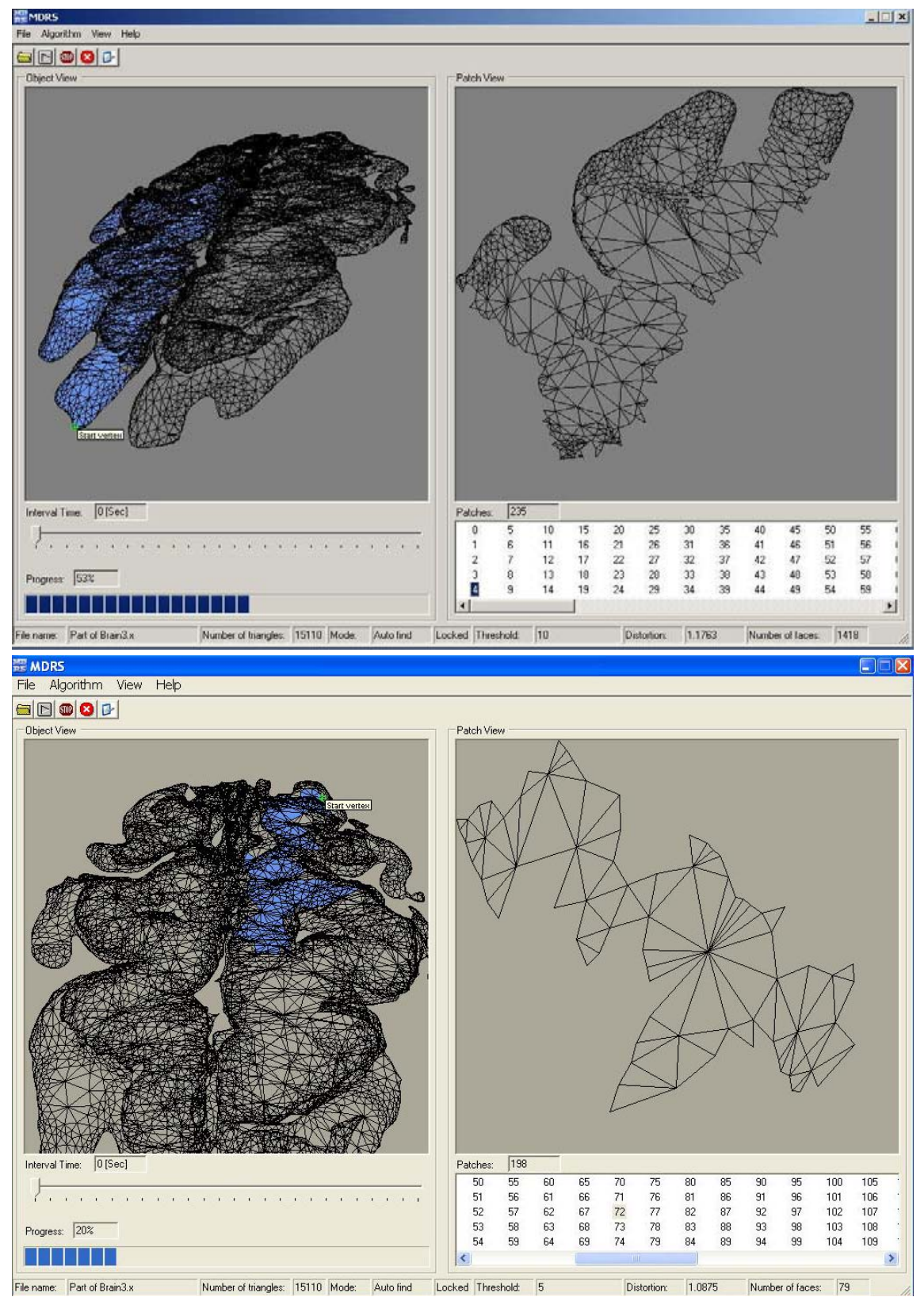

Fig. 9. Cerebral Cortex Flattening: Two patches obtained in the flattening of the parietal region. The resolution is 15.110 triangles, the angles chosen are $5^{\circ}$ and $10^{\circ}$, producing dilatations of 1.0875 and 1.1763 , respectively. 